\title{
Pervasive Crowd Mapping for Dynamic Environments
}

\author{
Hugo Paredes*, Hugo Fernandes*, Andre Sousa*, Fernando Koch ${ }^{\dagger}$, Vitor Filipe* and João Barroso* \\ *INESC TEC and UTAD, Portugal \\ \{hparedes,hugof,andresousa,vfilipe,jbarroso\}@utad.pt \\ ${ }^{\dagger}$ SAMSUNG Research Institute Brazil, Brazil \\ fernando.koch@samsung.com
}

\begin{abstract}
There is a demand for new models of computation intelligence for the recognition of the environment and the obstacles in each moment and the sharing of this information with other users providing temporary dangers notifications, which can enhance blind navigation experience and autonomy. We identified an opportunity to contribute with an integrated strategy to develop a solution to improve the blind autonomy and quality of life. We are looking for solutions to problems that have emerged from the accumulated experience in blind navigation systems research. The main objective of this paper is to present a conceptual model that works based on data obtained from sensors on passive monitoring, worn by bystanders that can combine and correlate the inference patterns that match the obstacles and/or dangers. The model has retro-feedback mechanisms, allowing the sensors to search and pervasively validate the existence of obstacle, ensuring the temporary basis of these risks.
\end{abstract}

\section{INTRODUCTION}

According to ITU, in 2015, 3.2 billion people, almost $44 \%$ of the world's population, are online. Moreover, the same source predicts 7 billion mobile cellular subscriptions by the end of 2015 , corresponding to a penetration rate of $97 \%$. Our everyday was hacked by devices and sensors carried everywhere and constantly acquiring and sending data in an always-connected environment. The Internet is filled with "things", connected and able to share data. Nowadays, the flow of people and devices provided with sensors allow data capture without user intervention raising many challenges and opportunities. Each "thing" should not be seen in a detached way, but as a collaborative agent that is a huge data gathering and labor force. Moreover, technological pervasive solutions can be embedded in usual online activities to enhance this distributed labor force.

People with disabilities represent specific subgroups in which particular requirements are intensified and therefore the impact of technology is most noticeable in their daily life. The blind, representing $4 \%$ of the world population, have their autonomy constrained by the sight of limitations with restrictions of environment perception, orientation and navigation. They often use the white cane for obstacle detection but in a new, unfamiliar setting, they require an accurate description of the surrounding environment. However, temporary obstacles and urban barriers represent a danger to the blind, even in wellknown environments. Traditional navigation and orientation aids are not accurate for all kind of obstacles. Therefore, the recognition of the environment and the obstacles in each moment and sharing of this information with other users providing temporary dangers notifications can enhance blind navigation experience and their autonomy.

An integrated strategy to develop a solution to improve the blind autonomy and quality of life is required, exploring existing solutions to problems that have emerged from the blind navigation systems research. This paper proposes a computational model of obstacle detection to be used in recommendation systems running on mobile computing' aiming to improve the quality of life of the blind population. The Pervasive Crowd Mapping for Dynamic Environments (PCM4DE) model operates around a combination of machine learning techniques and crowdsourcing mechanisms to collect data that can be combined to identify temporary obstacles or dangers in the environment, and that they can be spread to other users for its usage and confirm its sojourn, feeding back the system and ensuring its dynamism, precision, confidence and accuracy. The model resorts to data obtained from sensors on passive monitoring, worn by bystanders that can be combined and correlated to infer patterns that match the obstacles and/or dangers. Moreover, PCM4DE defines retro-feedback mechanisms, allowing the sensors/devices to search and validate the existence/persistence of obstacle with or without user interaction, ensuring the temporary basis of these risks.

The structure of the remaining paper is as follows: Section 2 describes background and related work. In section 3, the PCM4DE model is presented. The fourth section discusses the model, comparing it to current solutions, followed by some final remarks on Section 5.

\section{BACKGROUND}

In 2014 the World Health Organisation ${ }^{1}$ estimated that 285 million people worldwide are visually impaired representing $4 \%$ of the population. Sight loss is closely related to old age. Age-related blindness is increasing throughout the world, as is blindness due to uncontrolled diabetes. One in three senior citizens over 65 faces sight loss and $82 \%$ of blind people are over 50 years of age ${ }^{2}$. Moreover, according to the Population

\footnotetext{
${ }^{1}$ http://www.who.int/mediacentre/factsheets/fs282/en/

${ }^{2}$ http://www.euroblind.org/resources/information/
} 
Reference Bureau ${ }^{3}$ nearly 25 percent of people in the European Union in 2030 will be above age 65 .

Assistive technologies can provide a remarkable autonomy to the blind. Recently, computer vision systems have been developed to extract information about the surrounding environment and provide guidance for blind users [1], [2]. Multisensory approaches are also used to provide orientation and mobility to the blind [3], [4], addressing user location, navigation and environment recognition problems in a integrated solution [5]-[8]. The multi-sensory technology and data fusion is used in other contexts for the same purpose, as for instance in autonomous driving [9], [10]. Are often used data mining techniques [11], [12], hierarchical Markov decision processes [13] and neural networks [14], [15] for information extraction from the data gathered. However, most of them fail in assisting pedestrian navigation, especially for the blind, because they try to adapt vehicle navigation techniques.

Human-powered services can provide a complementary help needed for filling these gaps. Collaborative accessibility has been seen as a critical approach to enhance assistive technologies [16]. Design issues, cognitive barriers, accessibility problems, and accurate metadata authoring are some variables that can be analysed though these strategies [17]. Community services, as Be My Eyes ${ }^{4}$ allow volunteers all over the world to help blinds through a live video stream using their mobile phones. Mobile computing in a ubiquitous ecosystem presents several opportunities for crowd participation, adjusted to solve problems in the social inclusion and accessibility domain. In the last years some solutions of crowd participation in Smart Cities and Accessibility have been explored [18], [19]. These solutions are based on collecting smartphone data, store the data on a server and use models to identify problems in the city and their impact [20], [21]. This information can be used to build accessibility maps, and to define an appropriate action plan to address the detected issues, optimising resource allocation [22] and help people in their everyday life.

Recently the strategies of participation have migrated to a sensory participation using mobile devices [23], combining data obtained by sensing, crowdsourcing and mashing-up with main geo-referenced social systems to provide urban and architectural accessibility maps [24].

The PCM4DE follows this emerging research perspective of combining the Internet of Thing and Crowdsourcing strategies to enhance current state of the art in assistive technology. The proposal fills some of the current research gaps and has an important social impact.

Mobility is a major restriction for blind autonomy. Urban environments represent many dangers for blinds and current assistive technologies cannot provide enough feedback about the surrounding environment. Moreover, the environment is constantly changing, with new obstacles, barriers and dangers that are often temporary. An efficient solution that can recognise and warn about these dangers is required to enhance blind

\footnotetext{
${ }^{3} \mathrm{http}: / /$ goo.gl/zX6E4A

${ }^{4} \mathrm{http} / / /$ www.bemyeyes.org/
}

autonomy. Moreover, with the generalised and ubiquitous use of technology in everyday life, situational induced impairment and disability often occurs, as for example while reading an email or sending an SMS while walking in a sidewalk being temporarily "blind". A universal access solution that can avoid accidents in these kinds of situations would not only help blind users, but everyone.

\section{THE PCM4DE SYSTEM}

Assistive technologies play an important role in the lives of many people by providing the means to perform their daily living activities and helping people with disabilities to achieve greater independence and enhance their quality of life. In the last decades, addressing the challenging features and requirements of blind navigation has been a research hot topic. The redundancy of the information and location sources using active and passive sensors, the sensing of the users' surroundings using computer vision, the interaction with the user and his/her safety have been some of the prominent themes. Nowadays, in the navigation and orientation tasks one of the key issues is the decision-making capacity, and therefore, these support systems may be considered as decision support systems. As a decision support system, the data available and its accuracy and reliability determines the limit of the system capacity. One perspective into optimising such a system is through the enhancement of the available data.

An enhanced navigation solution for the blind in a city would aim to:

(i) map safe routes for people with accessibility issues;

(ii) inform about the proximity of problematic points;

(iii) recommend alternative routes when the proximity of trouble spots is detected.

Therefore, a solution requires the collection and processing of situational data as geotagged reports as the first part of the process.

The proposed solution explores the usage of data collected from the Internet of Things through multiple sensors that people (blind or not) wear in their everyday lives. A mobile application that collects data from the walking behaviour of the user in the sidewalk; the smart glasses that take pictures of the environment, capturing temporary obstacles; the microphone of the smart watch that listens to the whimpers of the user while crossing with a car parked on the sidewalk; the heart rate monitor that register the variations of heart rate while experiencing these emotions. These are just some examples of data that can be gathered. When considering public places frequented by hundreds of people, the same data can be grabbed by many users and be accurately associated to a temporary obstacle, danger or accessibility barrier. For this association a process of data fusion and information extraction is required. For enhancing the accuracy levels of the system, the information extracted can be made available for the users/sensors, in a retro-feedback validation process. In this process the sensors will be expecting to recognise the obstacle and notify the user. If the obstacle is not present they will register its absence, by sensing or by user interaction. In 
some particular cases the retro-feedback can explore the usage of traditional crowdsourcing strategies, requesting users simple interactions to recognised data gathered by the sensors, as a picture or a sound. The retro-feedback gathered data would be used to evolve the system and enhance the accuracy of the information extraction process, in a cyclic evolving process. The process is summarised in Fig. 1.

\section{A. Crowd participation strategies}

Currently there are numerous strategies to participate in crowdsourcing activities involving different mechanisms from the award of financial rewards to social rewards. One of the strategies that had higher success relies on gamification: to use the principles of games and socialisation to motivate individuals to participate in community tasks. An exhaustive survey of the existing strategies and its classification was required to identify the most relevant in each context and community. The analysis showed that the evaluation of each application using technological demonstrators is needed to understand the most relevant strategies, according to the classification followed and the community habits and requirements.

Another issue of this study is related with the mode of participation. Unlike traditional models, where there is an explicit participation, in this model the contribution is through the data captured by the users' sensors for joint computation. It must therefore be taken into account how to involve people at some specific locations and with sensing devices available (e.g., the camera of a smart phone) in supplying information about specific situations occurring around them (e.g., taking a picture of the location and making it available [24]-[27]). The data anonymization and privacy are main issues that must be clear to the users. Users' participation relies on their trust on the system and therefore the processing and usage of the data collected.

The PCM4DE System was designed in order to support multiple participation strategies. This flexibility enables the exploration of the participation strategies in different context, its classification according to a defined taxonomy and it inclusion as a model extension according to the specified requirements. Therefore, the implementations of the model can select the most relevant strategies to be implemented according to the specific community profile.

\section{B. Pervasive environmental data collection}

The use of sensors by users in their daily lives, without this interfering in their routines, is a premisse for the pervasive gathering of information. Collecting data from sensors is the starting point for the process of recognition of obstacles and hazards. Currently there are numerous types of sensors and devices that can be used by users, or embedded in their everyday devices, including smartphones, smart watches or smart glasses. On the other side there are environmental sensors that can also interact with user devices and provide data. Therefore, there is the need to use a taxonomy for classifying sensors [28]. A common taxonomy used for wireless micro-sensor is presented by Sameer et al. [29]. This taxonomy was used as a basis for classification of sensors and usage in the data collection module of the model.

Each sensor has the ability to collect data and send them. The volume of data generated by each sensor varies by the sampling frequency and the data collected. Many of the collected data can be discarded at the source, by duplication or the possibility of reconstruction with reduced computational weight, minimising the amount of stored data, filtering the data collected, avoiding the data meshing and optimising the data fusion and information extraction. But this process must be grounded and structured in order to ensure there are not discarded data that can be key in the decision making process. A second step of the process includes the analysis of data obtained by the selected sensors and the application of an optimised acquisition strategy.

\section{Data fusion and information extraction}

The definition of the data fusion process was grounded in the identification of data patterns, that were collected from data gathered from the sensors and the associated with obstacles and dangers in the environment. This identification took into consideration four types of situations: data from only one sensor that identifies an obstacle unambiguously; a sensor data that identifes an obstacle and can be complemented and enhanced in its accuracy with data from other sensors; data from various sensors that are essential for the unequivocal identification of an obstacle; and data from various sensors that are essential for the identification of an obstacle and can be supplemented and enhanced in its accuracy with data from other sensors.

The processing of the gathered data from sensors to identify objects or dangers for blinds is followed by a data fusion process for identification/inference of obstacles and dangers. The fusion of sensor data for the navigation and orientation has been subject of research for decades, as previously described. The most common techniques used are decision trees, data mining and Markov models. The model allows the usage of any of these techniques in order to efficiently support each situation and the comparison of results from the application of the different techniques. Thus it is possible to select a technique that best fits the situation. The identified obstacles and dangers are classified so that they can be added to the layers of the geographical information system supporting the blind navigation system and spread by its users. Associated with the classification information metadata is added indicating that it is a temporary obstacle/danger and its detection is being performed using the PCM4DE system. This information is essential to trigger the retro-feedback mechanisms explored in the sub-section.

\section{Retro-feedback mechanisms}

The retro-feedback mechanisms allow the validation and reevaluation of detected obstacle/dangers, ensuring the required dynamic data update in the geographic information systems. Moreover, asynchronous crowd evaluation mechanisms are triggered to validate the detection performed by 


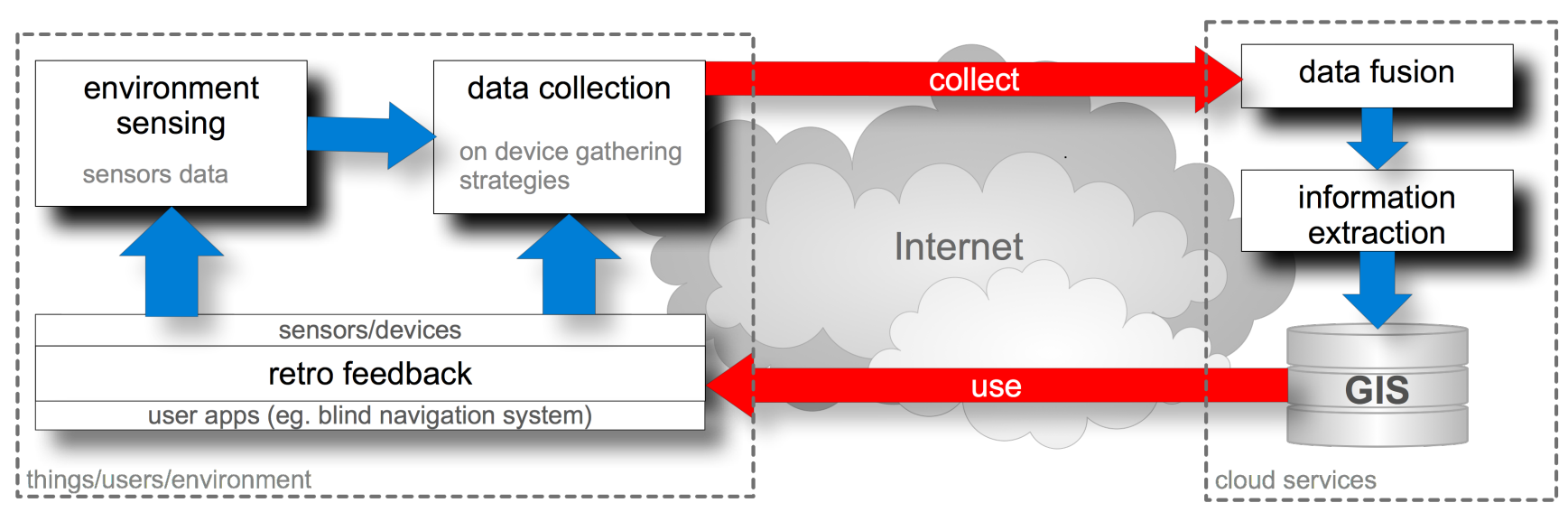

Fig. 1. Pervasive Crowd Mapping for Dynamic Environments conceptual diagram.

the system and provide feedback for the refinement of the detection mechanisms.

The development and implementation of retro-feedback mechanisms interrelates information processing of pervasive environmental data collection and data fusion and information extraction modules. These mechanisms are triggered in the data processing, when new information is added to the system by recognising a new obstacle/danger. This information is broadcast by the system and received by devices and sensors connected. Being an obstacle/danger associated with a geographic location, the sensors and connected devices will be expecting to find this element as part of the environment in that specific location. In case of detection/validation of the existence of the obstacle/danger different situations may occur:

- The user is interacting with a device and able to participate actively in the process;

- The validation/detection is passive, in pervasive mode, and the sensors are responsible for the capture of validation/detection data:

- The sensors provide the same kind of data, that was originally used to identify the obstacle;

- There are different sensors that can complement inferred information with complementary data;

Through these scenarios necessary data is collected to retrofeed the system for a reassessment of the environment. These mechanisms, their specification and formalisation is held in this module, for the evolution of the system and continuously adapting to new environmental conditions, which (by nature) are dynamic. In addition, a traditional crowdsourcing system can be used in particular cases for validation of the identifications made by the system with users. The gathered data results are used to feed back the detection mechanisms used and improve results. Another process associated with this module is the re-evaluation of the mechanisms used in the detection of obstacles/dangers taking into account the feedback data produced by assessing its behaviour and evolution.

\section{DISCUSSION}

Urban environments represent many dangers for the blind and current assistive technologies cannot provide enough feedback about the surrounding environment. In a constantly changing environment, obstacles, barriers and dangers are mostly temporary. An efficient and accurate solution that can recognise and warn about these temporary dangers, and follow the dynamic nature of the environment, is required to enhance blind autonomy.

The PCM4DE follows emerging research perspective of combining the Internet of Things and crowdsourcing to detect temporary obstacles. The strategies of participation have migrated to a sensory participation using mobile devices [23], combining data obtained by sensing, crowdsourcing and mashing-up with main geo-referenced social systems to provide urban and architectural accessibility maps [30]. However, these strategies only provide solutions for long term obstacles, failing to dynamicly adapt to the rapid changes in the environment. This is also a gap in previous solutions, as: in crowd participation solutions for Smart Cities and Accessibility [18], [19]; in the identification of problems in the city and their impact [20], [21]; and in building accessibility maps optimising resource allocation [22] and helping people in their everyday life.

A common problem in blind navigation systems is the close research to vehicle navigation. Multi-sensory technology and data fusion is used in autonomous driving [9], [10] to extract information about the surrounding environment and provide guidance. Data mining techniques [11], [12], hierarchical Markov decision processes [13] and neural networks [14], [15] are also often used for this purpose. However the types of obstacles and resources available in a vehicle are different from the ones worn by a person, requiring evaluation of the suitability of the techniques and mechanisms used.

The use of multi-sensory approaches to extract information about the surrounding environment and blind mobility [3], [4] are limited to address user location, navigation and partial environment recognition [5]-[8]. Moreover, most of 
these approaches rely on computer vision systems to extract surrounding information [1], [2]. The data fusion techniques and combination data gathered from multiple sensors have new perspectives with the Internet of Thing.

The PCM4DE proposal fills some of these research gaps. Moreover, as a generic model, it can also be used in other application scenarios: a core solution for environment description in augmented reality applications; a game context for user participation in urban planning; a stolen car finder.

\section{FINAL REMARKS}

This paper introduces a new perspective to enhance blind navigation systems with the usage of Internet of Thing.

The major tangible outcome of this paper is the model for identification of obstacles/dangers in the environment using the Internet of Things for gathering sensor data and crowdsourcing strategies for its evaluation and retro-feedback. Following the proposed technical approach, each module of the PCM4DE represents individual tangible outcomes (deliverables) that contributs to the general outcome.

The usage and integration of emerging technologies for the detection of obstacles, combining the Internet of Things, crowdsourcing and methods for artificial intelligence is a contribution as an intangible outcome that aims the enhancement of blind autonomy and minimisation of exposure to environmental temporary obstacles, urban barriers and dangers. Since this paper presents a conceptual model that yearns this major objective, at this phase it is expected to achieve a preliminary assessment of the feasibility of the proposed technology to solve the problem.

Beyond these contributions, it is expected that the implementation of a proof of concept of the PCM4DE model produces results and impacts into related scientific areas, and related with the presented challenges, namely: Internet of Things, universal access, social inclusion and collaboration.

\section{ACKNOWLEDGMENT}

This work is financed by the FCT - Fundação para a Ciência e a Tecnologia (Portuguese Foundation for Science and Technology) within project UID/EEA/50014/2013 and research grants SFRH/BD/89759/2012 and SFRH/BD/87259/2012.

\section{REFERENCES}

[1] M. Capp and P. Picton, "The optophone: an electronic blind aid," Engineering Science \& Education Journal, vol. 9, no. 3, pp. 137-143, 2000.

[2] C. ANCUTI, C. Ancuti, and P. Bekaert, "Colenvison: Color enhanced visual sonifier a polyphonic audio texture and salient scene analysis," 2009.

[3] S. Wang, H. Pan, C. Zhang, and Y. Tian, "Rgb-d image-based detection of stairs, pedestrian crosswalks and traffic signs," Journal of Visual Communication and Image Representation, vol. 25, no. 2, pp. 263-272, 2014.

[4] S. Alghamdi, R. van Schyndel, and I. Khalil, "Accurate positioning using long range active rfid technology to assist visually impaired people," Journal of Network and Computer Applications, vol. 41, pp. 135-147, 2014.

[5] S. Kammoun, G. Parseihian, O. Gutierrez, A. Brilhault, A. Serpa, M. Raynal, B. Oriola, M.-M. Macé, M. Auvray, M. Denis et al., "Navigation and space perception assistance for the visually impaired: The navig project," Irbm, vol. 33, no. 2, pp. 182-189, 2012.
[6] J. Faria, S. Lopes, H. Fernandes, P. Martins, and J. Barroso, "Electronic white cane for blind people navigation assistance," in World Automation Congress (WAC), 2010. IEEE, 2010, pp. 1-7.

[7] T. Adao, L. Magalhães, H. Fernandes, H. Paredes, and J. Barroso, "Navigation module of blavigator prototype," in World Automation Congress (WAC), 2012. IEEE, 2012, pp. 1-6.

[8] H. Fernandes, N. Conceição, H. Paredes, A. Pereira, P. Araújo, and J. Barroso, "Providing accessibility to blind people using gis," Universal Access in the Information Society, vol. 11, no. 4, pp. 399-407, 2012.

[9] D. Ferguson, M. Darms, C. Urmson, and S. Kolski, "Detection, prediction, and avoidance of dynamic obstacles in urban environments," in Intelligent Vehicles Symposium, 2008 IEEE. IEEE, 2008, pp. 11491154.

[10] K. Schueler, T. Weiherer, E. Bouzouraa, and U. Hofmann, "360 degree multi sensor fusion for static and dynamic obstacles," in Intelligent Vehicles Symposium (IV), 2012 IEEE. IEEE, 2012, pp. 692-697.

[11] C. Xianyi, J. Zhigang, and L. Jia, "Research on data fusion and data mining technology and its application in wireless sensor network," in Intelligent Networks and Intelligent Systems (ICINIS), 2012 Fifth International Conference on. IEEE, 2012, pp. 134-137.

[12] J. Liu, Z. Yan, and L. T. Yang, "Fusion-an aide to data mining in internet of things," Information Fusion, vol. 23, pp. 1-2, 2015.

[13] D. Akselrod, A. Sinha, and T. Kirubarajan, "Hierarchical markov decision processes based distributed data fusion and collaborative sensor management for multitarget multisensor tracking applications," in Systems, Man and Cybernetics, 2007. ISIC. IEEE International Conference on. IEEE, 2007, pp. 157-164.

[14] I. L. Davis, "Sensor fusion for autonomous outdoor navigation using neural networks," DTIC Document, Tech. Rep., 1995.

[15] F. Pacifici, F. D. Frate, W. J. Emery, P. Gamba, and J. Chanussot, "Urban mapping using coarse sar and optical data: Outcome of the 2007 grss data fusion contest," Geoscience and Remote Sensing Letters, IEEE, vol. 5, no. 3, pp. 331-335, 2008.

[16] H. Takagi, S. Kawanaka, M. Kobayashi, D. Sato, and C. Asakawa, "Collaborative web accessibility improvement: challenges and possibilities," in Proceedings of the 11th international ACM SIGACCESS conference on Computers and accessibility. ACM, 2009, pp. 195-202.

[17] H. Takagi, S. Kawanaka, M. Kobayashi, T. Itoh, and C. Asakawa, "Social accessibility: achieving accessibility through collaborative metadata authoring," in Proceedings of the 10th international ACM SIGACCESS conference on Computers and accessibility. ACM, 2008, pp. 193-200.

[18] D. Schuurman, B. Baccarne, L. De Marez, and P. Mechant, "Smart ideas for smart cities: investigating crowdsourcing for generating and selecting ideas for ict innovation in a city context," Journal of theoretical and applied electronic commerce research, vol. 7, no. 3, pp. 49-62, 2012.

[19] J. Eskelinen, A. Garcia Robles, I. Lindy, J. Marsh, and A. MuenteKunigami, "Citizen-driven innovation," 2015.

[20] C. Cardonha, D. Gallo, P. Avegliano, R. Herrmann, F. Koch, and S. Borger, "A crowdsourcing platform for the construction of accessibility maps," in Proceedings of the 10th International Cross-Disciplinary Conference on Web Accessibility. ACM, 2013, p. 26.

[21] F. Koch, C. Cardonha, J. M. Gentil, and S. Borger, "A platform for citizen sensing in sentient cities," in Citizen in Sensor Networks. Springer, 2013, pp. 57-66.

[22] K. Shigeno, S. Borger, D. Gallo, R. Herrmann, M. Molinaro, C. Cardonha, F. Koch, and P. Avegliano, "Citizen sensing for collaborative construction of accessibility maps," in Proceedings of the 10th International Cross-Disciplinary Conference on Web Accessibility. ACM, 2013, p. 24

[23] B. Guo, Z. Yu, X. Zhou, and D. Zhang, "From participatory sensing to mobile crowd sensing," in Pervasive Computing and Communications Workshops (PERCOM Workshops), 2014 IEEE International Conference on. IEEE, 2014, pp. 593-598.

[24] F. Alt, A. S. Shirazi, A. Schmidt, U. Kramer, and Z. Nawaz, "Locationbased crowdsourcing: extending crowdsourcing to the real world," in Proceedings of the 6th Nordic Conference on Human-Computer Interaction: Extending Boundaries. ACM, 2010, pp. 13-22.

[25] S. Reddy, D. Estrin, and M. Srivastava, "Recruitment framework for participatory sensing data collections," in Pervasive Computing. Springer, 2010, pp. 138-155.

[26] W. Willett, P. Aoki, N. Kumar, S. Subramanian, and A. Woodruff, "Common sense community: scaffolding mobile sensing and analysis for novice users," in Pervasive Computing. Springer, 2010, pp. 301318. 
[27] T. Yan, V. Kumar, and D. Ganesan, "Crowdsearch: exploiting crowds for accurate real-time image search on mobile phones," in Proceedings of the 8th international conference on Mobile systems, applications, and services. ACM, 2010, pp. 77-90.

[28] J. Gubbi, R. Buyya, S. Marusic, and M. Palaniswami, "Internet of things (iot): A vision, architectural elements, and future directions," Future Generation Computer Systems, vol. 29, no. 7, pp. 1645-1660, 2013.

[29] S. Tilak, N. B. Abu-Ghazaleh, and W. Heinzelman, "A taxonomy of wireless micro-sensor network models," SIGMOBILE Mob. Comput. Commun. Rev., vol. 6, no. 2, pp. 28-36, Apr. 2002. [Online]. Available: http://doi.acm.org/10.1145/565702.565708

[30] C. Prandi, P. Salomoni, and S. Mirri, "mpass: Integrating people sensing and crowdsourcing to map urban accessibility," in Proceedings of the IEEE International Conference on Consumer Communications and Networking Conference, 2014, pp. 10-13. 